SINP-2002-11/695

\title{
Near-horizon behavior of string-loop-corrected dyonic black holes
}

\author{
Mikhail Z. Iofa ${ }^{1}$ \\ Skobeltsyn Institute of Nuclear Physics \\ Moscow State University \\ Moscow 119992, Russia
}

December 24, 2018

\begin{abstract}
Dyonic black holes with string-loop corrections are studied in the near-horizon region. In perturbative heterotic string theory compactified to four dimensions with $N=2$ supersymmetry, in the first order in string-loop expansion parameter, we solve the system of Maxwell and Killing spinor equations for dyonic black hole. At the horizon, the stringloop-corrected solution displays restoration of spontaneously broken supersymmetry.
\end{abstract}

PACS: 04.70.Dy,04.50.+h,11.25.Db,11.25.Mj

Keywords: string theory, black holes, $\mathrm{N}=2$ supergravity

\footnotetext{
${ }^{1}$ Skobeltsyn Institute of Nuclear Physics, Moscow State University, Moscow 119992, Russia, e-mail: iofa@theory.sinp.msu.ru
} 
Since appearance of papers [1], where it was noted that the Robertson-Bertotti geometry is maximally supersymmetric, i.e. does not break any of supersymmetries of $N=2, d=4$ supergravity, there appeared a number of papers discussing this and related phenomena in different systems $[2,3,4,5]$.

In particular, it was made clear that if at special points of space-time the metric of a system is asymptotic to the Robertson-Bertotti metric, than at these points some partially broken supersymmetries can restore.

This property of the metrics imply the universality properties of black holes in supersymmetric theories: the entropy-area formula, attractor properties of a system at the points of supersymmetry restoration, etc. $[4,5,6,7,8,9,10,11,12,13]$ and refs. therein.

In this letter we discuss these properties in the case of the string-loop-corrected dyonic black hole. In the framework of perturbative heterotic string theory compactified to $4 D$ with $N=2$ supersymmetry, the prepotential of the effective low-energy theory (so-called STU model) receives only one-string-loop correction from string world sheets of torus topology $[14,15,16]$. String-loop expansion parameter is $\epsilon=e^{\phi_{\infty}}$, where $\phi_{\infty}=\left.\lim \phi\right|_{r \rightarrow \infty}$. Starting from the loopcorrected prepotential, in the first order in $\epsilon$, we solve Killing spinor equations (conditions for supersymmetry variations of spinors to vanish). In the region of small $r$, near the horizon of the black hole, we obtain expressions for the metric and moduli and verify vanishing of certain objects implying restoration of supersymmetry.

$N=1$ supersymmetric $6 D$ effective action of the heterotic string theory compactified to $4 D$ on a two-torus $T^{2}$ yields $N=2$ locally supersymmetric theory. Bosonic part of the $4 D$ effective action in the Einstein frame is [17]

$$
I_{4}=\int d^{4} x \sqrt{-g}\left[R-\frac{1}{2}(\partial \phi)^{2}-\frac{e^{-\phi}}{4} \mathcal{F}(L M L) \mathcal{F}+\frac{a_{1}}{4 \sqrt{-g}} \mathcal{F} L^{*} \mathcal{F}+\frac{1}{8} \operatorname{Tr}(\partial M L \partial M L)\right] .
$$

On the other hand, the bosonic part of the action of a $N=2$ locally supersymmetric theory has the form $[18,19,20]$

$$
I_{4}^{N=2}=\int d^{4} x \sqrt{-g}\left[\frac{1}{2} R+\left(\bar{N}_{I J} \mathcal{F}^{-I} \mathcal{F}^{-J}-N_{I J} \mathcal{F}^{+I} \mathcal{F}^{+J}\right)+k_{i \bar{j}} \partial_{\mu} z^{i} \partial^{\mu} \bar{z}^{j}+\ldots\right] .
$$

Here $\mathcal{F}_{\mu \nu}^{ \pm}=\frac{1}{2}\left(\mathcal{F}_{\mu \nu} \pm i \sqrt{-g} \mathcal{F}_{\mu \nu}\right)$, where ${ }^{*} \mathcal{F}_{\mu \nu}=\frac{1}{2} e_{\mu \nu \rho \lambda} \mathcal{F}^{\rho \lambda}$, and $e_{\mu \nu \rho \lambda}$ is the flat antisymmetric tensor, $e_{0123}=-1$. The couplings $N_{I J}$ are defined below, $k_{i \bar{j}}$ is the Kähler metric $k_{i \bar{j}}=\frac{\partial^{2} K}{\partial z^{i} \partial \bar{z}^{j}}$, where $K$ is the Kähler potential. The tree-level moduli are

$$
\begin{gathered}
\frac{X^{1}}{X^{0}}=z^{1}=i y_{1}=i\left(e^{-\phi}+i a_{1}\right), \\
\frac{X^{2}}{X^{0}}=z^{2}=i y_{2}=i\left(e^{\gamma+\sigma}+i a_{2}\right),
\end{gathered}
$$




$$
\frac{X^{3}}{X^{0}}=z^{3}=i y_{3}=i\left(e^{\gamma-\sigma}+i a_{3}\right)
$$

Here $\phi$ and $a_{1}$ are dilaton and axion, $a_{2}=B_{12}$, other moduli are identified by comparison with the metric of the two-torus

$$
G_{m n}=e^{2 \sigma}\left(\begin{array}{cc}
e^{2 \gamma-2 \sigma}+a_{3}^{2} & -a_{3} \\
-a_{3} & 1
\end{array}\right)
$$

In the holomorphic section which admits introduction of the prepotential, the effective action (1) can be identified with the action (2) having the prepotential of the STU model (for instance, [15])

$$
F=-\frac{X^{1} X^{2} X^{3}}{X^{0}}-i \epsilon X^{0^{2}} h\left(-i \frac{X^{2}}{X^{0}},-i \frac{X^{3}}{X^{0}}\right)+\ldots
$$

At the one-loop level dilaton mixes with other moduli and

$$
y_{1}=e^{-\phi}-\epsilon \frac{V}{2}+i a_{1}
$$

The Kähler potential is given by

$$
K=-\ln \left[\left(y_{1}+\bar{y}_{1}+\epsilon V\right)\left(y_{2}+\bar{y}_{2}\right)\left(y_{3}+\bar{y}_{3}\right)\right],
$$

and is invariant under symplectic transformations. Here $V$ is the Green-Schwarz function $V$ $[15,21]$

$$
V\left(y_{2}, \bar{y}_{2}, y_{3}, \bar{y}_{3}\right)=\frac{\operatorname{Re} h-\operatorname{Re} y_{2} \operatorname{Re} \partial_{y_{2}} h-\operatorname{Re} y_{3} \operatorname{Re} \partial_{y_{3}} h}{\operatorname{Re} y_{2} \operatorname{Re} y_{3}} .
$$

In the section with the prepotential the gauge couplings are calculated using the formula (for instance, $[18,19,20])$

$$
N_{I J}=\bar{F}_{I J}+2 i \frac{\left(I m F_{I K} X^{K}\right)\left(I m F_{J L} X^{J}\right)}{\left(X^{I} I m F_{I J} X^{J}\right)}
$$

where $F_{I}=\partial_{X^{I}} F, F_{I J}=\partial_{X^{I} X^{J}}^{2} F$, etc.

In the case of black hole solutions in which we are interested in this paper, the tree-level moduli $y_{i}$ are real. Since the expressions of the first order in string coupling are calculated by substituting for the arguments the tree-level moduli, below, in cases where this does not lead to confusion, we use the same notation $y_{i}$ for the real parts of the moduli. Imaginary parts of the moduli corresponding to dyonic solution may appear in the first order in string coupling. 
In the first order in string coupling constant, the gauge couplings $N_{I J}$ are [22]

$$
\begin{aligned}
& N_{00}=i y^{3}\left(-1+\epsilon \frac{n}{4 y^{3}}\right), \quad N_{01}=-\epsilon \frac{n+2 v}{4 y_{1}}-i \epsilon a_{1} \frac{y_{2} y_{3}}{y_{1}}, \\
& N_{02}=-\epsilon \frac{n+2 v-2 y_{2} h y+4 y_{2} h_{2}}{4 y_{2}}-i \epsilon a_{2} \frac{y_{1} y_{3}}{y_{2}}, \\
& N_{03}=-\epsilon \frac{n+2 v+2 y_{3} h y+4 y_{3} h_{3}}{4 y_{3}}-i \epsilon a_{3} \frac{y_{1} y_{2}}{y_{3}}, \\
& N_{11}=-i \frac{y^{3}}{y_{1}^{2}}\left(1+\epsilon \frac{n}{4 y^{3}}\right), \quad N_{12}=i y_{3} \epsilon \frac{2 y_{2} h y-n}{4 y^{3}}+\epsilon a_{3}, \\
& N_{13}=i y_{2} \epsilon \frac{2 y_{3} h y-n}{4 y^{3}}+\epsilon a_{2}, \quad N_{23}=i y_{1} \epsilon \frac{2 y h y-4 y_{2} h_{23} y_{3}-n}{4 y^{3}}+\epsilon a_{1}, \\
& N_{22}=-i \frac{y^{3}}{y_{2}^{2}}\left(1-\epsilon \frac{y_{2} h_{23} y_{3}}{y^{3}}+\epsilon \frac{n}{4 y^{3}}\right), \quad N_{33}=-i \frac{y^{3}}{y_{3}^{2}}\left(1-\epsilon \frac{y_{3} h_{23} y_{3}}{y^{3}}+\epsilon \frac{n}{4 y^{3}}\right) .
\end{aligned}
$$

Here we introduced the notations: $y^{3}=y_{1} y_{2} y_{3}, h y=h_{a} y_{a}=h_{2} y_{2}+h_{3} y_{3}, h_{a}=\partial_{y_{a}} h, h_{a b}=$ $\partial_{y_{a}} \partial_{y_{b}} h$ and

$$
v=h-y_{a} h_{a}, \quad n=h-h_{a} y_{a}+y_{a} h_{a b} y_{b}, \quad y_{2} h y=y_{2} h_{2 a} y_{a} .
$$

The field equations and the Bianchi identities for the gauge field strengths are

$$
\begin{aligned}
\partial_{\mu}\left(\sqrt{-g} \operatorname{Im} G_{I}^{-\mu \nu}\right) & =0 \\
\partial_{\mu}\left(\sqrt{-g} \operatorname{Im} \mathcal{F}^{-J \mu \nu}\right) & =0
\end{aligned}
$$

where $G_{I}^{-\mu \nu}=\bar{N}_{I J} \mathcal{F}^{-J \mu \nu}$. In sections which do not admit a prepotential (including that which naturally appears in compactification of the heterotic string action), the gauge couplings are obtained by making a symplectic transformation of the couplings calculated in the section with the prepotential

$$
\hat{N}=(C+D N)(A+B N)^{-1} .
$$

In the first order in string coupling, transformation from the section with the prepotential to that associated with the heterotic string compactification is performed by symplectic transformation with the matrices

$$
\begin{array}{ll}
A=\operatorname{diag}(1,0,1,1)+\epsilon\left(a_{i j}\right), & B=\operatorname{diag}(0,1,0,0)+\epsilon\left(b_{i j}\right), \\
C=\operatorname{diag}(0,1,0,0)+\epsilon\left(c_{i j}\right), & D=\operatorname{diag}(1,0,1,1)+\epsilon\left(d_{i j}\right),
\end{array}
$$


where $a, b, c$ and $d$ are constant symmetric matrices. The form of the corrections to the treelevel matrices [15] is constrained by the requirement that in the heterotic section the loop corrections to the couplings are proportional to $\epsilon e^{\phi}$. This results in symplectic transformation with $a=b=0, c$ is an arbitrary symmetric matrix with $c_{1 i}=0$ and the only non-zero element of the matrix $d$ is $d_{11}[22]$.

General dyonic solution can be obtained by solving the Killing spinor equations which are conditions for the supersymmetry transformations of the chiral gravitino $\psi_{\alpha \mu}$ and gaugini $\lambda^{i \alpha}$ to vanish (for instance, $[19,23,24]$ )

$$
\begin{aligned}
& \delta \psi_{\alpha \mu}=D_{\mu} \epsilon_{\alpha}-T_{\mu \nu}^{-} \gamma^{\nu} \epsilon_{\alpha \beta} \epsilon^{\beta}=0, \\
& \delta \lambda_{\alpha}^{i}=i \gamma^{\mu} \partial_{\mu} z^{i} \epsilon^{\alpha}+G_{\mu \nu}^{-i} \gamma^{\mu} \gamma^{\nu} \epsilon^{\alpha \beta} \epsilon_{\beta}=0,
\end{aligned}
$$

where

$$
D_{\mu} \epsilon_{\alpha}=\left(\partial_{\mu}-\frac{1}{4} w_{\mu}^{\hat{a} \hat{b}} \gamma_{\hat{a}} \gamma_{\hat{b}}+\frac{i}{2} Q_{\mu}\right) \epsilon_{\alpha}
$$

Here $w_{\mu}^{\hat{a} \hat{b}}$ and $Q_{\mu}$ are the spin and Kähler connections, and one introduces symplectic invariants $[19,25,10]$

$$
\begin{aligned}
& S_{\mu \nu}=X^{I} \operatorname{Im} N_{I J} \mathcal{F}_{\mu \nu}^{-J}, \quad T_{\mu \nu}^{-}=2 i e^{K / 2} S_{\mu \nu}, \\
& G_{\mu \nu}^{-i}=-k^{i \bar{j}} \bar{f}_{\bar{j}}^{I} \operatorname{Im} N_{I J} \mathcal{F}_{\mu \nu}^{-J} .
\end{aligned}
$$

Here $k^{i \bar{j}}$ is the inverse Kähler metric, and

$$
f_{i}^{I}=\left(\partial_{i}+\frac{1}{2} \partial_{i} K\right) e^{K / 2} X^{I}
$$

In the case of a stationary spherically-symmetric solutions with the metric

$$
d s^{2}=-e^{2 U(r)}\left(d t+w_{m}(r) x^{m}\right)^{2}+e^{-2 U(r)}\left(d r^{2}+r^{2} d \Omega^{2}\right)
$$

The $\mu=0$ component of the gravitini Killing spinor equation can be presented as

$$
w_{0}^{-\hat{a} \hat{b}} \gamma_{\hat{a}} \gamma_{\hat{b}} \epsilon_{\alpha}-e^{U} T_{0 n}^{-} \gamma_{\hat{n}} \epsilon_{\alpha \beta} \epsilon^{\beta}=0
$$

Here the indices with hats refer to the tangent space basis. Assuming the Ansatz for a constraint on the supersymmetry parameter $\epsilon^{\alpha}=\gamma_{\hat{0}} \epsilon^{\alpha \beta} \epsilon_{\beta}$ (cf. $\left.[24,10]\right)$, separating the coefficient at the spinor structure and taking the real part of the resulting equation, we have

$$
\frac{1}{4} \partial_{n} e^{2 U}-e^{U} \operatorname{Re} T_{0 n}^{-}=0
$$


where we used that $w_{0}^{\hat{0} \hat{b}}=\frac{1}{2} \partial_{b} e^{2 U}$. Substituting the Ansatz in gaugini Killing equation, we obtain

$$
\left(i e^{U} \partial_{n} z^{i}-4 G_{0 n}^{-i}\right) \gamma^{\hat{0}} \gamma^{\hat{n}} \epsilon^{\alpha \beta} \epsilon_{\beta}=0 .
$$

Contracting Eq.(22) with the functions $f_{i}^{I}$, we have

$$
i f_{i}^{I} \partial_{n} z^{i} e^{U}+4\left(\frac{1}{2} \mathcal{F}_{0 n}^{-I}+e^{K} \bar{X}^{I} S_{0 n}\right)=0 .
$$

Using the relations of special $N=2$ geometry, Eqs.(23) can be recast in the form which contains $G_{I 0 n}^{-}$and $\bar{F}_{I}$. Contracting Eq.(23) with $\bar{F}_{I}$ and using identities

$$
\mathcal{F}^{-I} \bar{F}_{I}=G_{I}^{-} \bar{X}^{I}, \quad \bar{F}_{I} f_{i}^{I}=\bar{X}^{I} g_{I i},
$$

where $g_{I i}=\left(\partial_{i}+\frac{1}{2} \partial_{i} K\right) e^{K / 2} F_{I}$, which follow from definitions of $f_{i}^{I}$ and $G_{I \mu \nu}$, we have

$$
\bar{X}^{I} g_{I i} \partial_{n} z^{i}+4 e^{-U}\left(\frac{1}{2} G_{I 0 n}^{-} \bar{X}^{I}+e^{K} \bar{F}_{I} \bar{X}^{I} S_{0 n}\right)=0
$$

Removing the functions $\bar{X}^{I}$, we obtain the symmetric equation

$$
g_{I i} \partial_{n} z^{i}+4 e^{-U}\left(\frac{1}{2} G_{-I 0 n}+e^{K} \bar{F}_{I} S_{0 n}\right)=0 .
$$

Substituting the gravitino equation, Eqs.(23) and (24) are presented as (cf. [11])

$$
\begin{gathered}
-2 \mathcal{F}_{0 n}^{-I}=i\left[e^{U} \partial_{n}\left(e^{K / 2} X^{I}\right)-\left(e^{K / 2} \bar{X}^{I}\right) \partial_{n} e^{U}\right]+2 \operatorname{Im} T_{0 n}^{-} e^{K / 2} \bar{X}^{I}-\operatorname{Im}\left(\partial_{i} K \partial_{n} y_{i}\right) e^{K / 2+U} X^{I}, \\
-2 G_{I 0 n}^{-}=i\left[e^{U} \partial_{n}\left(e^{K / 2} F_{I}\right)-\left(e^{K / 2} \bar{F}_{I}\right) \partial_{n} e^{U}\right]+2 \operatorname{Im} T_{0 n}^{-} e^{K / 2} \bar{F}_{I}-\operatorname{Im}\left(\partial_{i} K \partial_{n} y_{i}\right) e^{K / 2+U} F_{I} .
\end{gathered}
$$

Here we used the equality $\partial_{i} K \partial_{n} z^{i}=\frac{1}{2} \partial_{n} K+i \operatorname{Im}\left(\partial_{i} K \partial_{n} z^{i}\right)$. Eqs.(25) and (26) are not independent, but one set can be obtained from the other. One can also take some equations from the first set, and the remaining equations from the second.

Let us solve Eqs.(25) and (26). We consider a tree-level dyonic black hole solution with vanishing axionic parts, i.e. the tree-level moduli $y_{i}$ are real. Axions $a_{i}=\operatorname{Im} y_{i}$ can appear at higher orders in $\epsilon$. The holomorphic section associated with the heterotic string compactification is

$$
\left(\hat{X}^{I}, \hat{F}_{I}\right)=\left(1, y_{2} y_{3}, i y_{2}, i y_{3} ;-i y_{1} y_{2} y_{3},-i y_{1}, y_{1} y_{3}-\epsilon h_{2}, y_{1} y_{2}-\epsilon h_{3}\right) .
$$

The tree-level dyonic solution contains two magnetic and two electric fields with strengths

$$
* \hat{\mathcal{F}}_{0 r}^{0}=-\frac{P^{0}}{\sqrt{-g^{\prime}}}, \quad * \hat{\mathcal{F}}_{0 r}^{1}=-\frac{P^{1}}{\sqrt{-g^{\prime}}}
$$


and

$$
\hat{\mathcal{F}}_{0 r}^{2}=-\frac{Q_{2}}{\sqrt{-g^{\prime}} \operatorname{Im} \hat{N}_{22}}, \quad \hat{\mathcal{F}}_{0 r}^{3}=-\frac{Q_{3}}{\sqrt{-g^{\prime}} \operatorname{Im\hat {N}_{33}}},
$$

where $\sqrt{-g^{\prime}}=e^{-2 U} r^{2}$ and the gauge couplings are

$$
\hat{N}_{00}=-i y_{1} y_{2} y_{3}, \quad \hat{N}_{11}=-i \frac{y_{1}}{y_{2} y_{3}}, \quad \hat{N}_{22}=-i \frac{y_{1} y_{3}}{y_{2}}, \quad \hat{N}_{33}=-i \frac{y_{1} y_{2}}{y_{3}} .
$$

Since the tree-level moduli are real, $\operatorname{Im}\left(\partial_{i} K \partial_{n} z^{i}\right)=O\left(\epsilon^{2}\right)$. Taking imaginary parts of Eqs.(25) for $I=0,1$ and Eqs.(26) for $I=2,3$, we obtain

$$
\begin{aligned}
& \sqrt{-g} \hat{\mathcal{F}}_{0 n}^{I}=e^{2 U} \partial_{n}\left(e^{K / 2-U} \operatorname{Re} \hat{X}^{I}\right)+O\left(\epsilon^{2}\right), \\
& \sqrt{-g}^{*} \hat{G}_{I 0 n}=e^{2 U} \partial_{n}\left(e^{K / 2-U} \operatorname{Re} \hat{F}_{I}\right)+O\left(\epsilon^{2}\right) .
\end{aligned}
$$

Substituting (28) and (29) in the system (30) and (31), we obtain the equations

$$
-\frac{P^{0}}{r^{2}}=\left(e^{K / 2-U}\right)^{\prime}, \quad-\frac{P^{1}}{r^{2}}=\left(e^{K / 2-U} y_{2} y_{3}\right)^{\prime}
$$

and

$$
-\frac{Q_{2}}{r^{2}}=\left(e^{K / 2-U} y_{1} y_{3}\right)^{\prime}, \quad-\frac{Q_{3}}{r^{2}}=\left(e^{K / 2-U} y_{1} y_{2}\right)^{\prime}
$$

with the tree-level solutions (cf. [26, 27, 28, 10, 11, 13])

$$
\begin{aligned}
& e^{2 U_{0}}=\frac{r^{2}}{\left(H^{0} H^{1} H_{2} H_{3}\right)^{1 / 2}}, \quad y_{1(0)} \equiv e^{-\phi_{0}} \equiv f_{0}^{-1}=\left(\frac{H_{2} H_{3}}{H^{0} H^{1}}\right)^{1 / 2}, \\
& y_{2(0)} \equiv e^{\gamma_{0}+\sigma_{0}}=\left(\frac{H^{1} H_{3}}{H^{0} H_{2}}\right)^{1 / 2}, \quad y_{3(0)} \equiv e^{\gamma_{0}-\sigma_{0}}=\left(\frac{H^{1} H_{2}}{H^{0} H_{3}}\right)^{1 / 2} .
\end{aligned}
$$

where

$$
H^{0}=\sqrt{8} P^{0}+a r, \quad H^{1}=\sqrt{8} P^{1}+a^{-1} r, \quad H_{2}=\sqrt{8} Q_{2}+b r, \quad H_{3}=\sqrt{8} Q_{3}+b^{-1} r .
$$

The constants are constrained by the requirement that solution is asymptotically flat.

From the tree-level solutions it follows that expressions $\partial_{n} z^{i}$ are finite and the functions $G_{\mu \nu}^{-i}$ vanish in the limit $r \rightarrow 0$. Introducing new variable, $\rho=\frac{1}{r}$, one obtains a conformally flat form of the metric in the limit $\rho \rightarrow \infty$. As follows from the above, in this limit the derivatives of the moduli $\partial_{\rho} z^{i}$ vanish. Due to vanishing of the function $e^{U}$ in the limit $r \rightarrow 0$, all the terms in gravitini and gaugini equations vanish separately and yield no restrictions on supersymmetry parameter (cf. $[2,3,5]$ ), i.e. at this point supersymmetry is restored. 
In papers [27] it was shown that in general case of string tree-level dyonic black hole, nonzero total electric and magnetic charges yield constraints, each preserving $1 / 2$ of the supersymmetry unbroken (so that general dyonic configuration preserves $1 / 4$ of the supersymmetry unbroken). This result is not in contradiction with the restoration of supersymmetry at the horizon, because the constraints are obtained from Killing spinor equations which contain the metric component $e^{2 U}$. At the horizon, the terms multiplied by the metric component $e^{2 U}$ vanish, and there appear no relations leading to constraints.

Let us turn to the loop-corrected dyonic solution. The functions $\phi, \gamma$ and $\sigma$ which enter the moduli (3) and the function $2 U$ in metric are split into the tree-level parts $\phi_{0}, \gamma_{0}, \sigma_{0}, 2 U_{0}$ and the parts of the first order in string coupling: $\phi=\phi_{0}+\epsilon \phi_{1}, \ldots, 2 U=2 U_{0}+\epsilon u_{1}$. With the required accuracy, the Kähler potential is

$$
e^{K}=\frac{f_{0} e^{-2 \gamma_{0}}}{8}\left[1+\epsilon\left(\phi_{1}-2 \gamma_{1}\right)\right]
$$

In the first order in string coupling, taking into account that the terms of the main order in string coupling cancel, Eqs.(30) with $I=0,1$ yield

$$
\begin{aligned}
& \left(\phi_{1}-u_{1}-2 \gamma_{1}\right)^{\prime}+\frac{1}{2}\left(\phi_{0}-2 U_{0}-2 \gamma_{0}\right)^{\prime}\left(\phi_{1}-u_{1}-2 \gamma_{1}\right)=0 \\
& \left(\phi_{1}-u_{1}+2 \gamma_{1}\right)^{\prime}+\frac{1}{2}\left(\phi_{0}-2 U_{0}+2 \gamma_{0}\right)^{\prime}\left(\phi_{1}-u_{1}+2 \gamma_{1}\right)=0 .
\end{aligned}
$$

From this system we find that in the limit $r \rightarrow 0$ both functions $\gamma_{1}$ and $\phi_{1}-u_{1}$ vanish as $O(r)$. Substituting the loop-corrected expressions $\hat{F}_{2}=y_{1} y_{3}-\epsilon h_{2}$ and $\hat{F}_{3}=y_{1} y_{2}-\epsilon h_{3}$ and introducing $L_{2}=\epsilon h_{2} y_{2} e^{-2 \gamma_{0}}$ and $L_{3}=\epsilon h_{3} y_{3} e^{-2 \gamma_{0}}$, we reduce the second pair of equations (31) to the form

$$
\begin{gathered}
\left(\phi_{1}+u_{1}+2 \sigma_{1}+\left(V+2 L_{2}\right) f_{0}\right)^{\prime}-\frac{1}{2}\left(\sigma_{0}+U_{0}+\frac{\phi_{0}}{2}\right)^{\prime}\left(\phi_{1}+u_{1}+2 \sigma_{1}+\left(V+2 L_{2}\right) f_{0}\right)=0, \\
\left(\phi_{1}+u_{1}-2 \sigma_{1}+\left(V+2 L_{3}\right) f_{0}\right)^{\prime}-\frac{1}{2}\left(-\sigma_{0}+U_{0}+\frac{\phi_{0}}{2}\right)^{\prime}\left(\phi_{1}+u_{1}-2 \sigma_{1}+\left(V+2 L_{3}\right) f_{0}\right)=0 .
\end{gathered}
$$

From these equations, in the limit $r \rightarrow 0$ we find

$$
\phi_{1}=u_{1}=-H f_{0}(0)=-\left.\frac{h\left(y_{2(0)}, y_{3(0)}\right)}{2 y_{1(0)} y_{2(0)} y_{3(0)}}\right|_{r=0}=\left.\frac{h\left(y_{2(0)}, y_{3(0)}\right)}{2}\right|_{r=0}\left(\frac{P^{0^{3}}}{P^{1} Q_{2} Q_{3}}\right)^{1 / 2}
$$

and

$$
\sigma_{1}=\left.\frac{1}{2}\left(L_{3}-L_{2}\right)\right|_{r=0}\left(\frac{P^{0} P^{1}}{Q_{2} Q_{3}}\right)^{1 / 2}+O(r)
$$


Here we introduced

$$
H=\left.\frac{V+L}{2}\right|_{r=0}=\left.\frac{h\left(y_{2(0)}, y_{3(0)}\right)}{2 y_{2(0)} y_{3(0)}}\right|_{r=0}, \quad L=\frac{L_{2}+L_{3}}{2} .
$$

Let us consider solution of the gaugini Killing spinor equations written in an alternative form (23). At the tree level, substituting explicit expressions for the field strengths (28) and (29) and solutions for the moduli (35), we find that in the limit $r \rightarrow 0$ all the combinations $\left(\frac{1}{2} \hat{\mathcal{F}}_{0 n}^{-I}+e^{K} \overline{\hat{X}}^{I} S_{0 n}\right)$ vanish implying that $\left.\partial_{n} y_{i}\right|_{r \rightarrow 0}$, are finite and $G_{\mu \nu}^{i}$ vanish. Vanishing of of the tensor $G_{\mu \nu}^{i}$ together with Bertotti-Robinson form of the metric near the point $r=0$ ensure vanishing of the Weyl tensor and conformal invariance of the theory in this region.

Keeping the terms of the main and the first orders in string coupling, the Maxwell equations

$$
\partial_{\mu}\left(\sqrt{-g} \operatorname{Im} \hat{N}_{I J} \hat{\mathcal{F}}^{J}+\operatorname{Re} \hat{N}_{I J}{ }^{*} \hat{\mathcal{F}}^{J}\right)^{\mu \nu}=0 .
$$

written in the heterotic holomorphic section are

$$
\begin{gathered}
\partial_{r}\left[\sqrt{-g}\left(\operatorname{Im} \hat{N}_{00} \hat{\mathcal{F}}^{0}+\operatorname{Im} \hat{N}_{02} \hat{\mathcal{F}}^{2}+\operatorname{Im} \hat{N}_{03} \hat{\mathcal{F}}^{3}\right)+\operatorname{Re} \hat{N}_{00}{ }^{*} \hat{\mathcal{F}}^{0}+\operatorname{Re} \hat{N}_{01}{ }^{*} \hat{\mathcal{F}}^{1}\right]^{0 r}=0 \\
\partial_{r}\left[\sqrt{-g}\left(\operatorname{Im} \hat{N}_{11} \hat{\mathcal{F}}^{1}+\operatorname{Im} \hat{N}_{12} \hat{\mathcal{F}}^{2}+\operatorname{Im}_{13} \hat{\mathcal{F}}^{3}\right)+\operatorname{Re} \hat{N}_{10}{ }^{*} \hat{\mathcal{F}}^{0}+\operatorname{Re} \hat{N}_{11}{ }^{*} \hat{\mathcal{F}}^{1}\right]^{0 r}=0 \\
\partial_{r}\left[\sqrt{-g}\left(\operatorname{Im} \hat{N}_{22} \hat{\mathcal{F}}^{2}+\operatorname{Im} \hat{N}_{23} \hat{\mathcal{F}}^{3}\right)+\operatorname{Re} \hat{N}_{20}{ }^{*} \hat{\mathcal{F}}^{0}+\operatorname{Re} \hat{N}_{21}{ }^{*} \hat{\mathcal{F}}^{1}\right]^{0 r}=0 \\
\partial_{r}\left[\sqrt{-g}\left(\operatorname{Im} \hat{N}_{33} \hat{\mathcal{F}}^{2}+\operatorname{Im}_{32} \hat{\mathcal{F}}^{2}\right)+\operatorname{Re} \hat{N}_{30}{ }^{*} \hat{\mathcal{F}}^{0}+\operatorname{Re} \hat{N}_{31}{ }^{*} \hat{\mathcal{F}}^{1}\right]^{0 r}=0 .
\end{gathered}
$$

Only the diagonal gauge couplings $\hat{N}_{I I}$ contain the parts of the main order in string coupling. The field strengths $\hat{\mathcal{F}}_{0 r}^{0,1}$, absent at the tree level, are of the first order in string coupling. Solving the Maxwell equations in the holomorphic section associated with the heterotic string compactification with the gauge couplings $\hat{N}_{I J}$ calculated from the couplings (9) by using the transformations (13), (14), we obtain

$$
\begin{aligned}
\hat{\mathcal{F}}^{00 r} & =\frac{q_{0}-a_{1} P^{1}-a_{a} Q_{a}}{\sqrt{-g^{\prime}} \operatorname{Im} \hat{N}_{00}}, \\
\hat{\mathcal{F}}^{10 r} & =\frac{q_{1}-a_{1} P^{0}+a_{3} Q_{2} / y_{3}^{2}+a_{2} Q_{3} / y_{2}^{2}}{\sqrt{-g^{\prime}} \operatorname{Im} \hat{N}_{11}} \\
\hat{\mathcal{F}}^{20 r} & =\frac{Q_{2}-\operatorname{Re} \hat{N}_{20} P^{0}-\operatorname{Re} \hat{N}_{21} P^{1}-\frac{I m N_{23}}{I m N_{33}} Q_{3}}{\sqrt{-g^{\prime}} \operatorname{Im} \hat{N}_{22}}, \\
\hat{\mathcal{F}}^{30 r} & =\frac{Q_{3}-\operatorname{Re} \hat{N}_{30} P^{0}-\operatorname{Re} \hat{N}_{31} P^{1}-\frac{I m N_{32}}{I m N_{22}} Q_{2}}{\sqrt{-g^{\prime}} \operatorname{Im} N_{33}} .
\end{aligned}
$$


Substituting the field strengths (44)-(47), we have [22]

$$
\begin{aligned}
& S_{0 n}=\left\{\left[P^{0}\left(\operatorname{Im} N_{00}+y_{i} \operatorname{Re} N_{i 0}\right)-P^{1} y_{1}-\left(Q_{2} y_{2}+Q_{3} y_{3}\right)\right]\right. \\
& \left.\left.-i \epsilon\left[P^{0}\left(a_{1} y_{2} y_{3}+a_{2} y_{1} y_{3}+a_{3} y_{1} y_{2}\right)+a_{a} Q_{a}+a_{1} P^{1}-q_{0}-q_{1} y_{2} y_{3}\right)\right]\right\} \frac{i}{2} e^{2 U} \frac{x^{n}}{r^{3}}
\end{aligned}
$$

Because Killing spinor equations are linear in derivatives of the moduli, in the first order in string coupling the equations for the real and imaginary parts of the moduli decouple. To obtain the loop corrections to the dilaton $\phi$ and the metric component $e^{2 U}$ as well as to the metric of the two-torus $G_{m n}$ it is sufficient to solve the Killing spinor equations for the real parts of the moduli. At this stage, the imaginary parts of the moduli can be neglected.

The combinations $f_{i}^{I} \partial_{n} z^{i}$ which enter Eqs.(23) are

$$
f_{i}^{0} \partial_{n} z^{=} \frac{1}{2 \sqrt{8}} e^{1 / 2\left(\phi_{0}-2 \gamma_{0}\right)}\left(\phi_{0}^{\prime}-2 \gamma_{0}^{\prime}\right)\left[1+\epsilon \frac{\phi_{1}^{\prime}-2 \gamma_{1}^{\prime}}{\phi_{0}^{\prime}-2 \gamma_{0}^{\prime}}+\frac{\epsilon}{2}\left(\phi_{1}-2 \gamma_{1}\right)\right] \frac{x^{n}}{r}
$$

and

$$
f_{i}^{1} \partial_{n} z^{=} \frac{1}{2 \sqrt{8}} e^{1 / 2\left(\phi_{0}+2 \gamma_{0}\right)}\left(\phi_{0}^{\prime}+2 \gamma_{0}^{\prime}\right)\left[1+\epsilon \frac{\phi_{1}^{\prime}+2 \gamma_{1}^{\prime}}{\phi_{0}^{\prime}+2 \gamma_{0}^{\prime}}+\frac{\epsilon}{2}\left(\phi_{1}+2 \gamma_{1}\right)\right] \frac{x^{n}}{r} .
$$

Other combinations are $f_{n}^{i} \partial_{n} z^{i}=i y_{i}\left(f_{n}^{0}+e^{K / 2} \partial_{n} y_{a}\right)$, where $i=1,2,3$. The expression $e^{K} S_{0 n}$ in Eqs.(23) can be presented as ${ }^{2}$

$$
e^{K} S_{0 n}=\left(1-\epsilon \frac{V f_{0}}{2}\right)\left(S_{0}+\epsilon S_{1}\right) \frac{i}{16 \sqrt{-g^{\prime}}} \frac{x^{n}}{r}
$$

where

$$
S_{0}=P^{0}+P^{1} e^{-2 \gamma_{0}}+Q_{2} e^{\phi_{0}-\gamma_{0}+\sigma_{0}}+Q_{3} e^{\phi_{0}-\gamma_{0}-\sigma_{0}}
$$

and

$$
\begin{aligned}
& S_{1}=P^{0} 2(V+L) f_{0}+P^{1} e^{-2 \gamma_{0}}\left(-2 \gamma_{1}\right) \\
& +Q_{2} e^{\phi_{0}-\gamma_{0}+\sigma_{0}}\left(\phi_{1}-\gamma_{1}+\sigma_{1}+\frac{V f_{0}}{2}\right)+Q_{3} e^{\phi_{0}-\gamma_{0}-\sigma_{0}}\left(\phi_{1}-\gamma_{1}-\sigma_{1}+\frac{V f_{0}}{2}\right) .
\end{aligned}
$$

Substituting the above expressions, we obtain the gaugini Killing equations (23) with $I=0,1$ in the form

$$
\begin{array}{r}
\frac{\phi_{1}^{\prime}-2 \gamma_{1}^{\prime}}{\phi_{0}^{\prime}-2 \gamma_{0}^{\prime}}+\frac{1}{2}\left(\phi_{1}-2 \gamma_{1}-u_{1}\right)-\frac{S_{1}-S_{0} \frac{V f_{0}}{2}}{4 P^{0}-S_{0}}=0, \\
\frac{\phi_{1}^{\prime}+2 \gamma_{1}^{\prime}}{\phi_{0}^{\prime}+2 \gamma_{0}^{\prime}}+\frac{1}{2}\left(\phi_{1}+2 \gamma_{1}-u_{1}\right)-\frac{S_{1}-S_{0} \frac{V f_{0}}{2}}{4 P^{1} H^{0} / H^{1}-S_{0}}=0 .
\end{array}
$$

\footnotetext{
${ }^{2}$ More exactly, here we consider gaugini Killing spinor equations for the real parts of the moduli, i.e. for $S_{0 n}$ we take the first (real) term in curly brackets in (48). Imaginary parts of the moduli will be discussed separately.
} 
At small $r$ we have

$$
S_{0}=4 P^{0}+2 P^{0} r\left(\phi_{0}^{\prime}-2 \gamma_{0}^{\prime}\right)(0)+O\left(r^{2}\right)
$$

and

$$
S_{1}=2 P^{0}\left(\phi_{1}-2 \gamma_{1}+H f_{0}\right)(0)+O(r) .
$$

Eqs.(54) split into the following system

$$
\begin{array}{r}
\gamma_{1}^{\prime}+2 \gamma_{0}^{\prime}\left(\phi_{1}-u_{1}\right)+2 \phi_{0}^{\prime} \gamma_{1}=0, \\
\phi_{1}^{\prime}+\frac{\phi_{0}^{\prime}}{2}\left(\phi_{1}-u_{1}\right)+2 \gamma_{0}^{\prime} \gamma_{1}-\frac{S_{1}-S_{0} \frac{V f_{0}}{2}}{4 P^{0}-S_{0}}=0
\end{array}
$$

Substituting (55) and (56) and noting that the functions $\phi_{1}$ and $u_{1}$ are finite at the origin, and $\gamma_{1}$ is of order $O(r)$, at small $r$ we reduce the second equation (57) to the form

$$
\phi_{1}^{\prime}-\frac{\phi_{1}}{r}-\frac{H(0) f_{0}}{r}=0
$$

Similar transformations of the gravitini equation yield

$$
\frac{u_{1}^{\prime}}{2 U_{0}^{\prime}}-\frac{u_{1}}{2}+\frac{1}{2}\left(\phi_{1}-2 \gamma_{1}-u_{1}\right)-\frac{S_{1}-S_{0} \frac{V f_{0}}{2}}{4 P^{0}-S_{0}}=0,
$$

At small $r 2 U_{0}^{\prime}=\frac{2}{r}+O(1)$, and the gravitini Killing equation takes the same form as (58)

$$
u_{1}^{\prime}-\frac{u_{1}}{r}-\frac{H(0) f_{0}}{r}=0 .
$$

Solving Eqs.(58) and (60), we reproduce the solution (39). In the same way we obtain the above solutions for $\gamma_{1}$ and $\sigma_{1}$.

Let us consider the equations for imaginary parts $a_{i}$ of the moduli $y_{i}=\operatorname{Re} y_{i}+i a_{i}$. The functions $a_{i}$ are zero at the tree level, and can appear in the first order in string coupling. Below, in the equations for $a_{i}$, the moduli $R e y_{i}$ appear only in the main order, and again we keep notations $y_{i}$ for the real parts of the moduli. Contracting gaugini Killing Eq.(22) with the metric $k_{i \bar{j}}$, introducing $T_{I 0 r} \equiv \operatorname{Im} N_{I J} \mathcal{F}_{0 r}^{-J}$ and separating the imaginary part of the resulting equation, we have

$$
k_{\overline{j i}} a_{i}^{\prime}+4 e^{-U} \operatorname{Im}\left(\bar{f}_{\bar{j}}^{I} T_{I}\right)=0 .
$$

Introducing $\tilde{q}_{1}=q_{1} e^{2 \gamma_{0}},\left(y_{2(0)} y_{3(0)}=e^{2 \gamma_{0}}\right.$, see (35)) with the required accuracy, Eqs.(61) are written as

$$
a_{1}^{\prime}+\frac{4 e^{U+K / 2} y_{1}}{r^{2}}\left[q_{0}+\tilde{q}_{1}-a_{1} P^{1}-a_{a} Q_{a}+P^{0}\left(-a_{1} y_{2} y_{3}+a_{2} y_{1} y_{3}+a_{3} y_{1} y_{2}\right)\right]=0,
$$




$$
\begin{aligned}
& a_{2}^{\prime}+\frac{4 e^{U+K / 2} y_{2}}{r^{2}}\left[q_{0}-\tilde{q}_{1}-a_{1} P^{1}-a_{a} Q_{a}+\hat{P}^{0}\left(+a_{1} y_{2} y_{3}-a_{2} y_{1} y_{3}+a_{3} y_{1} y_{2}\right)\right]=0 \\
& a_{3}^{\prime}+\frac{4 e^{U+K / 2} y_{3}}{r^{2}}\left[q_{0}-\tilde{q}_{1}-a_{1} P^{1}-a_{a} Q_{a}+P^{0}\left(+a_{1} y_{2} y_{3}+a_{2} y_{1} y_{3}-a_{3} y_{1} y_{2}\right)\right]=0
\end{aligned}
$$

Here all the functions $y_{i}, U$ and $K$ are taken in the main order in string coupling. Let us introduce the functions $b_{i}=a_{i} / y_{i}$. All the coefficients at the functions $b_{i}$ in Eqs.(62) rewritten through the functions $b_{i}$ in the limit $r=0$ coincide and are equal to $\left(\frac{P^{1} Q_{2} Q_{3}}{P^{0}}\right)^{1 / 2} \equiv \alpha$, and the expression $\frac{4 e^{U+K / 2}}{r^{2}}$ at small $r$ is $\frac{\sqrt{2}}{r \alpha}$. In the limit of small $r$, we obtain the system $(62)$ in the form

$$
\begin{aligned}
& b_{1}^{\prime}+b_{1} \frac{y_{1}^{\prime}}{y_{1}}+\frac{\sqrt{2}}{r \alpha}\left[q_{0}+\tilde{q}_{1}-2 \alpha b_{1}+O(r)\right]=0, \\
& b_{2}^{\prime}+b_{2} \frac{y_{2}^{\prime}}{y_{2}}+\frac{\sqrt{2}}{r \alpha}\left[q_{0}-\tilde{q}_{1}-2 \alpha b_{2}+O(r)\right]=0, \\
& b_{3}^{\prime}+b_{3} \frac{y_{3}^{\prime}}{y_{3}}+\frac{\sqrt{2}}{r \alpha}\left[q_{0}-\tilde{q}_{1}-2 \alpha b_{3}+O(r)\right]=0 .
\end{aligned}
$$

with a solution

$$
\begin{aligned}
& b_{1}=\frac{q_{0}+\tilde{q}_{1}}{2 \alpha}+c_{1} r+O\left(r^{2}\right), \\
& b_{a}=\frac{q_{0}-\tilde{q}_{1}}{2 \alpha}+c_{a} r+O\left(r^{2}\right), \quad a=2,3 .
\end{aligned}
$$

where $c_{i}$ are arbitrary constants.

Using the above asymptotics of the axions, we can consider stationarity properties of the loop-corrected solution. Taking the imaginary part of the gravitini spinor Killing equation (20), we have

$$
\frac{1}{2} * w_{0}^{\hat{0} \hat{n}} \gamma^{\hat{0}} \gamma^{\hat{n}} \epsilon_{\alpha}+e^{U} \operatorname{Im} T_{0 n}^{-} \gamma^{\hat{0}} \gamma^{\hat{n}} \epsilon_{\alpha \beta} \epsilon^{\beta}=0
$$

where ${ }^{*} w_{0}^{\hat{0} \hat{n}}=\frac{1}{2} e^{\hat{n} \hat{p} \hat{q}}\left(\partial_{p} w_{q}-\partial_{q} w_{p}\right)$ and $\operatorname{Im} T_{0 n}^{-}=2 R e e^{K / 2} S_{0 n}$ with $S_{0 n}$ from (48). In the limit of small $r$ we have $\operatorname{Im} T=O\left(r^{0}\right)$ and $e^{U} \operatorname{Im} T=O(r)$ implying vanishing of the functions $w_{n}$ in this limit and stationarity of solution. Also, suitably adjusting the free constants in (64), the asymptotic (physical) charges of the electric fields $\hat{\mathcal{F}}^{0}$ and $\hat{\mathcal{F}}^{1}$ can be made vanishing.

To conclude, general loop-corrected dilatonic black holes with four charges, as the treelevel solutions, at the horizon display restoration of $N=2$ supersymmetry. The metric in the 
near-horizon region becomes of the Robinson-Bertotti type. This result is natural, provided perturbation theory does not violate supersymmetry.

\section{Acknowledgments}

This work was partially supported by the RFFR grant No 02-02-16444.

\section{References}

[1] G. W. Gibbons and C. M. Hull, Phys. Lett. 109B (1982) 190, G. W. Gibbons, in Supersymmetry, Supergravity and Related Topics, eds. F. del Aguila et al (World Scientific, Singapore 1985).

[2] R. Kallosh, Phys. Lett. B282 (1992) 80, hep-th/9201029.

[3] R. Kallosh and A.Peet, Phys. Rev. D46 (1992) 5223, hep-th/9209116.

[4] R. Kallosh et. al., Phys. Rev. D46 (1992) 5278, hep-th/9205027.

[5] S. Ferrara and R. Kallosh, Phys. Rev. D54 (1996) 1514, 1525 hep-th/9602136;9603090.

[6] S. Ferrara, R. Kallosh and A. Strominger, Phys. Rev. D52 (1995) 5412, hep-th/9508072.

[7] E. Bergshoeff, R. Kallosh and T. Ortin, Nucl. Phys. B478 (1996) 156, hep-th/9605059.

[8] K. Behrndt et. al., Phys. Rev. D54, 6293 (1996), hep-th/9608059.

[9] K. Behrndt et. al., Nucl. Phys. B488 (1997) 236, hep-th/9610105.

[10] K. Behrndt, D. Lust and W. A. Sabra, Nucl. Phys. B510 (1998) 266, hep-th/9705169.

[11] G. L. Cardoso, B. de Wit, J. Käppeli and T. Mohaupt, JHEP 0012 (2000) 019, hepth/0009234.

[12] T. Mohaupt, Fortsch. Phys. 49 (2001) 3, hep-th/0009234.

[13] M. Bertolini and M. Trigiante, Int. J. Mod. Phys. A15 (2000) 5017, hep-th/9910237; Nucl. Phys. B582 (2000) 393, hep-th/0002191.

[14] I. Antoniadis et.al., Nucl. Phys. B447 (1995) 35, hep-th/9504034.

[15] B. de Wit, V. Kaplunovsky, J. Louis and D. Luest, Nucl. Phys. B451 (1995) 53, hepth/9504006. 
[16] J. A. Harvey and G. Moore, Nucl. Phys. B463 (1996) 315, hep-th/9510182.

[17] A. Sen, Int. J. Mod. Phys. A9 (1994) 3707, hep-th/9402002.

[18] B. de Wit and A. Van Proeyen, Nucl. Phys. B245 (1984) 89; B. de Wit, P. Lauwers and A. Van Proeyen, Nucl. Phys. B255 (1985) 569.

[19] L. Andrianopoli et. al. , J. Geom. Phys. 23 (1997) 111, hep-th/9605032.

[20] A. Ceresole, R. D'Auria, S. Ferrara and A. Van Proeyen, Nucl. Phys. B444 (1995) 92, hep-th/9502072.

[21] E. Kiritsis, C. Kounnas, P.M. Petropoulos and J. Rizos, Nucl. Phys B483 (1997) 141, hep-th/9608034.

[22] M. Z. Iofa, hep-th/0203046; Mod. Phys. Lett. A17 (2002) 355, hep-th/0104163.

[23] A. Strominger, Comm. Math. Phys. 133, 163 (1990).

[24] P. Fre, Nucl. Phys. Proc. Suppl. 57 (1997) 52, hep-th/9701054.

[25] A. Ceresole, R. D'Auria and S. Ferrara, Proc. of ICTP Trieste Conf." S-duality and Mirror Symmetry" (1995), hep-th 9509160.

[26] M. Cvetič and D. Youm, Nucl. Phys. B453 (1995) 259, hep-th/9505045; Phys. Rev. D53 (1996) 584, hep-th/9507090.

[27] M. Cvetič and D. Youm, Phys. Rev. D53 (1996) 584, hep-th/9507090, Nucl.Phys. B438 (1995) 182, Addendum-ibid. B449 (1995) 146, hep-th/9409119.

[28] M. Cvetič and A. A. Tseytlin, Phys. Lett. B366 (1996) 95, hep-th/9510097; Phys. Rev. D53 (1996) 5619, hep-th/9512031. 